\title{
HIV ASSOCIATED LYMPHOMA - TWO CASE REPORT SERIES
}

\author{
Assist. Prof. Liana-Cătălina Gavriliu, $\mathrm{MD}, \mathbf{P h D}^{1,2}$, \\ Assoc. Prof. Otilia-Elisabeta Benea, $\mathrm{MD}, \mathbf{P h D}^{1,2}$ \\ 1 "Carol Davila" University of Medicine and Farmacy, Bucharest \\ 2"Prof. Dr. Matei Bals" National Institute of Infectious Disease, Bucharest
}

\begin{abstract}
Combined antiretroviral therapy significantly improved the prognosis and the life expectancy of HIV patients. Along with this, the profile of HIV associated malignant pathologies has changed. Among these, lymphoma, with a higher incidence than in general population, are an important cause of mortality and morbidity in HIV infected people.

We present two cases of HIV-associated lymphoma. In the first case, a newly HIV diagnosed patient, with a good immunological status, developed non-Hodgkin's Burkitt-type lymphoma and he had a fulminant evolution towards death. In the second case, a patient known for over twenty years with HIV infection and who had discordant immunological and virusological response to antiretroviral therapy, was diagnosed with Hodgkin's lymphoma and he had a favorable evolution with chemotherapy. Both patients required exhaustive medical investigations and a close interdisciplinary cooperation during the entire time of diagnostic and therapeutic procedures.
\end{abstract}

Keywords: HIV, non-Hodgkin lymphoma, Hodgkin lymphoma

\section{BACKGROUND}

Combined antiretroviral therapy (CART) significantly improved the prognosis and life expectancy of HIV positive patients. However, while improving viral control, a change of malignancies panel associated with HIV infection was recorded. Kaposi sarcoma, an AIDS defining illness, has been undergoing a significantly decrease in incidence. Among malignant nonHodgkin lymphoma (NHL) the same overall tendency was observed, with some differences between subtypes: a decreasing rate of diffuse large B cell lymphoma (DLBCL), primary CNS lymphoma, plasmablastic lymphoma, primary effusion lymphoma and a stable rate of Burkitt lymphoma. Other oncologic diseases such as solid tumors and Hodgkin Lymphoma (HL), a non-defining AIDS malignancy, retained a stable incidence or showed an increased incidence in patients with HIV infection after CART introduction $(1,2)$. However, while CART brought benefits on the immune status and control of viral replication, the incidence of lymphomas among these patients remained higher than in general population. Thus, HL is 5-15 times more frequent and NHL is 60 to 200 times more frequent among patients with HIV infection $(1,3,4)$ compared to general population $(5,6)$.

Patients are usually diagnosed in a late-stage of the hematologic illness, with extranodal involvement and B signs of disease (fever, weight loss, profuse night sweats).

Corresponding author:

Liana-Cătălina Gavriliu, "Prof. Dr. Matei Bals" National Institute of Infectious Disease, 1 Calistrat Grozovici Street, District 2, Bucharest

E-mail: lianagavriliu@yahoo.com 
Prior to CART, the evolution under specific treatment of HIV patients with lymphoma was unfavorable, grafted by multiple complications and opportunistic infections development. CART allowed immunologic control and determined a better response to chemotherapy, with improved overall survival $(7,8)$.

We present two cases of lymphoma associated with HIV infection. In the first case, a newlydiagnosed HIV patient having a good immunological status was also diagnosed with Burkitt type non-Hodgkin lymphoma, with a rapid evolution towards death. In the second case, the patient with over two decades history of HIV infection with a discordant immunologic and virologic response to antiretroviral therapy was diagnosed with Hodgkin lymphoma, until now having a favorable outcome undergoing chemotherapy.

\section{CASE PRESENTATIONS}

\section{Patient 1}

A 27 years old male patient presented to our clinic in February 2014 with symptoms started two months prior, associating multiple enlarged lymph nodes with rapidly marked growth, low back pain radiating towards lower limbs, abdominal pain and diplopia. Previously to his presentation in our clinic, the patient was hospitalized in a medical oncology unit, where he underwent lymph node biopsy and histopathological examination which revealed granulomatous lymphadenitis.

The patient mentioned discordant HIV serology two years previously.

Physical examination on admission to our clinic revealed a pale, febrile patient $\left(38^{\circ} \mathrm{C}\right)$, with tachycardia (100 bpm), $\mathrm{BP}=110 / 70 \mathrm{mmHg}$, multiple lymphadenopathies measuring 2-3 cm in laterocervical, submandibular, supraclavicular, axillary, inguinal regions, right axilary lymph nodes mass of $16-17 \mathrm{~cm}$ in diameter with anterior thoracic wall extension, right ptosis, hepat- osplenomegaly (lower liver egde and spleen palpable $3 \mathrm{~cm}$ and $4 \mathrm{~cm}$, respectively below costal margins). Neurological examination identified III,IV,VI left cranial nerves paralysis (ptosis and fixed pupil), hyposensitivity in the teritory of the $V$ left cranial nerve and right mandibular branch, XII left cranial nerve paresis, tetraparesis affecting predominantly the lower limbs, positive bilateral Babinski sign. The rest of clinical examination was normal.

Laboratory tests on admission showed 7140 leukocytes/mmc (41.8\% lymphocytes, 43.1\% neutrophils, $6.7 \%$ monocytes, $8.4 \%$ eosinophils), normochromic normocytic anemia (10.4 $\mathrm{g} / \mathrm{dl}$ ), platelets $183000 / \mathrm{mmc}, \mathrm{ESR}=90 \mathrm{~mm} / 1 \mathrm{~h}$, $\mathrm{CRP}=192 \mathrm{mg} / \mathrm{l}$, negative procalcitonin, nitrogen retention (creatinine $=1.7 \mathrm{mg} / \mathrm{dl}$ ), normal liver enzymes, significantly increased LDH (4810 U/l), increased uric acid (145 mg/dl). Blood cultures were collected and the results were negative after 7 days.

HIV infection was confirmed: positive antiHIV $1+2$ antibodies (two samples), positive Wester-Blott HIV1. Immunologic and virologic evaluation revealed CD4 lymphocites count of 442/mmc and HIV-RNA = $193522 \mathrm{copies} / \mathrm{ml}$. Virological profile was completed with: Toxoplasma gondii antibodies (IgM and IgG negative), CMV antibodies (IgM negative IgG positive), Epstein-Barr virus (IgM negative, IgG antibodies not available), negative HBsAg, positive HBs antibodies, negative VHC antibodies. Quantiferon TB test was positive.

Lymph node biopsy was performed again. Histopathology examination revealed adipose connective tissue with malignant medium cell proliferation, with frequent mitosis and apoptosis and with a high mitotic index; frequent macrophages that formed prominent "starry-sky" appearance. Immunohistochemistry analysis (IHC) showed positive L26/CD20 cells, negative TdT, positive Ki67 in 95\% of the tumor cells, diffuse positive $\mathrm{CD} 10$, negative $\mathrm{Bcl} 2$, negative $\mathrm{CD} 3$ in tumor cells, positive in small reactive lymphocytes, 
positive Bcl6, weak positive CD79a in tumor cells, diffuse positive PAX5, negative MPO. Histopathological and IHC appearance was compatible with unclassifiable B-cell NHL, with intermediate aspects between diffuse large B-cell NHL and Burkitt lymphoma. Hematological evaluation was completed with bone marrow biopsy. Morphological analysis showed an extensive cell density, $85 \%$ blasts, most of them of medium size, with low cytoplasm with intense basophilia and cytoplasmic vacuoles. IHC examination revealed diffuse marrow involvement and confirmed the diagnosis of Burkitt medium cell NHL. (Fig. 1, 2, 3). Bacteriological tests performed on lymph node fragment and on bone marrow aspirate were negative for bacterial, fungal and tuberculosis etiology.

Thoracic CT scan (Fig. 4) showed a right axillary mass of $121 / 113 \mathrm{~mm}$ in axial dimensions
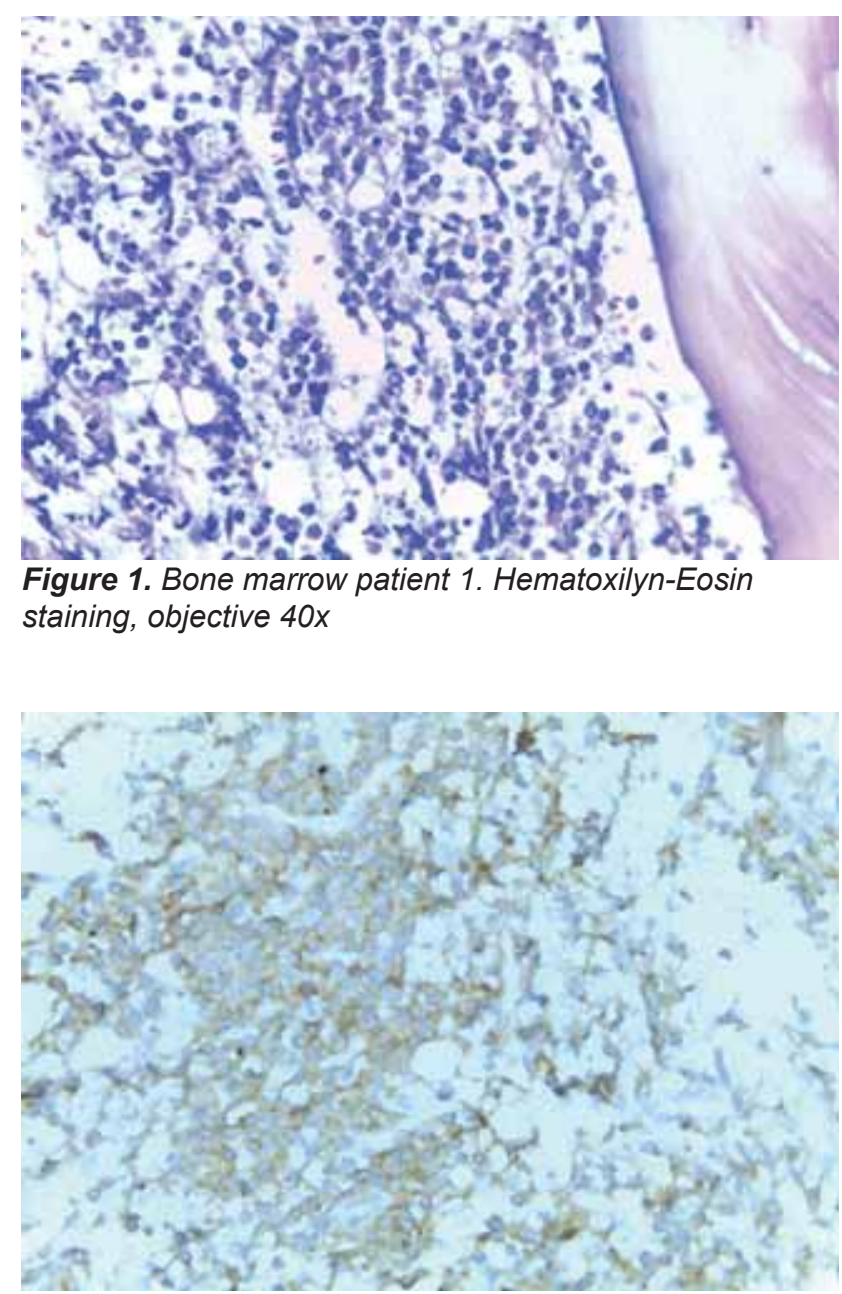

Figure 2. Immunohistochemistry CD10 bone marrow patient 1 , objective $40 x$

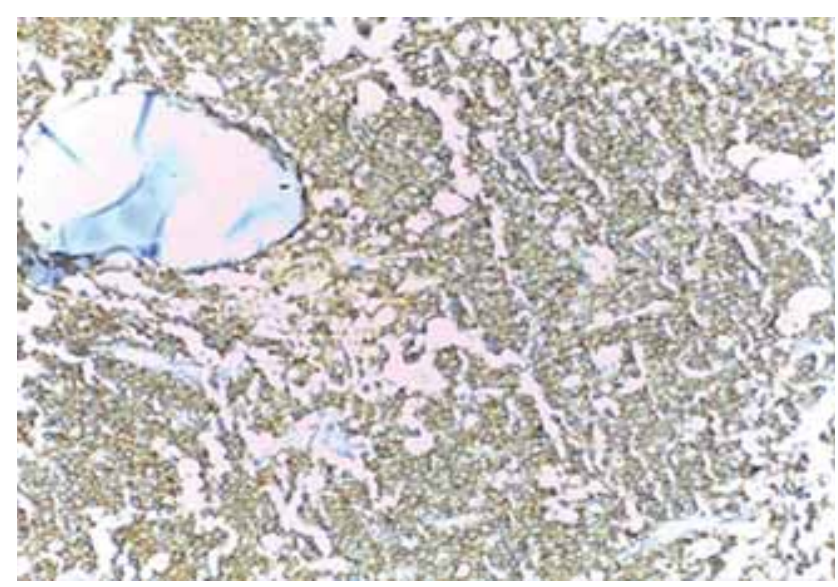

Figure 3. Immunohistochemistry CD20 bone marrow patient 1 , objective $40 x$

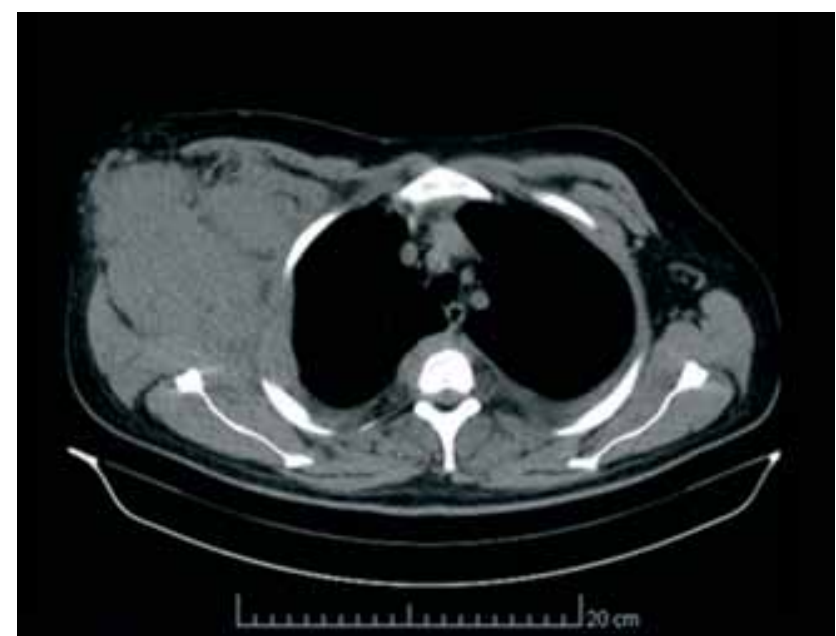

Figure 4. Thoracic CT scan patient 1. Right axillary tumoral mass. Many similar other lesions were found in the thoracic wall, near the ribs, especially on the left and along the spine.

and $136 \mathrm{~mm}$ in cranio-caudal diameter, without contrast uptake, formed by confluence of several lesions. Lesions with similar characteristics were found in the thoracic wall, in the contiguity of left anterior II rib arch, III bilateral, IV and V left, VI bilateral and VIII and IX lateral part of ribs arches, without cortical bone involvement. Multiple thoracic paravertebral and anterolateral masses from T1-T10 with maximum dimensions of $38 / 22 \mathrm{~mm}$, also without cortical bone destruction, were observed. There were also revealed small bilateral pleural effusions, with a maximum thickness of about $20 \mathrm{~mm}$ on the left side and $18 \mathrm{~mm}$ on the right side.

Cerebral MRI (Fig. 5) showed infiltrative lesions in the clivus disrupting the cortical bone and with extension in the posterior retroclival 


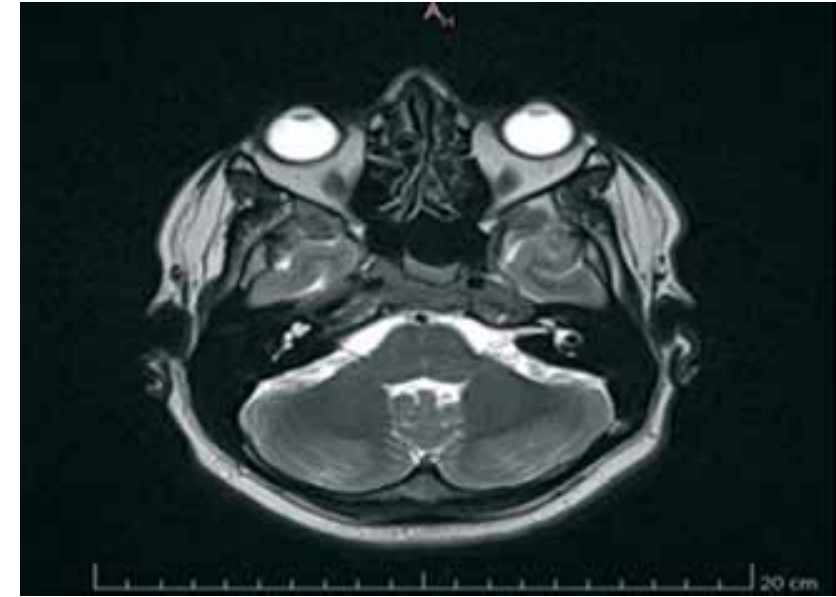

Figure 5. Brain MRI patient 1. Infiltrative lesion of the clivus

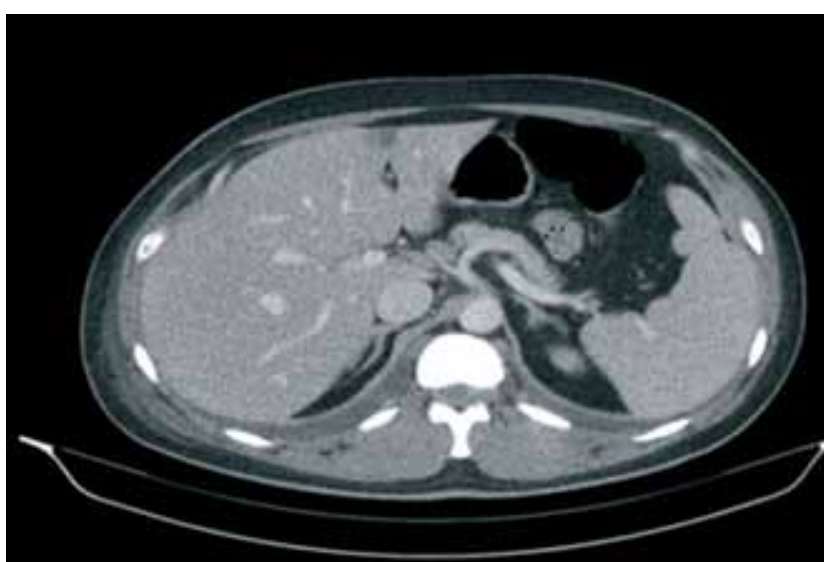

Figure 6. Abdominal CT scan patient 1. Nodular image in the VI segment in the right hepatic lobe, nodular image into the lateral abdominal wall, next to VI hepatic segment

region, anterior to sphenoidal sinus and superior intraselar, moving the pituitary gland to the top and lateral, filling bilaterally the Meckel cavum, with infiltration of the cavernous sinus and occupying left hypoglossal canal. The lesion enclosed the right VI cranial nerve, infiltrated the cisternal region of $\mathrm{V}$ cranial nerve and moved the III left cranial nerve laterally before its entrance into the cavernous left sinus.

Abdominal ultrasound and CT scan (Fig. 6) revealed hepatomegaly $(17.5 \mathrm{~cm})$, splenomegaly $(15 / 5.5 \mathrm{~cm})$, nodular image of $10 \mathrm{~mm}$ in the VI segment of the right hepatic lobe and $4.5 / 1.5 \mathrm{~cm}$ image into the lateral abdominal wall, next to VI hepatic segment.

During the 14 days of hospitalization, the patient received corticosteroid therapy (Methylprednisolone $32 \mathrm{mg} /$ day) combined with $\mathrm{H} 2$ receptor antagonist (ranitidine), anti-pyretic and substitution therapy with blood transfusions. Initially there were taken into consideration, besides lymphoma, tuberculosis and systemic fungal infection. The patient was started on tuberculosis treatment (isoniazid, rifampicin, pyrazinamide, etambutol) from $4^{\text {th }}$ day of hospitalization until the $14^{\text {th }}$ day. After obtaining all histopathological and bacteriological results the decision of discontinuing this treatment was made. The same applied for antifungal therapy (amphotericin B). After confirming the HIV infection, antiretroviral therapy was started on the $11^{\text {th }}$ day (Raltegravir, Emtricitabine, Tenofovir).

The outcome was unfavorable, with fever persistence, general deterioration, increase of neurological signs, intensification of anemia, thrombocytopenia and elevation of LDH.

On the $14^{\text {th }}$ day, the histopathological and IHC results were available, which certified the Burkitt type NHL diagnosis. The patient was transferred to the hematology clinic in order to start the chemotherapeutic treatment. 48 hours later the patient died.

\section{Patient 2}

A 27 years old male patient was addmited to our clinic in February 2016 for marked asthenia, profuse sweating, unproductive cough, started about three weeks earlier. The patient was diagnosed with HIV infection at the age of 3. Over the years he underwent several ART protocols (Table 1), lately recording discordant immunovirological response (Table 2). His medical history included pulmonary tuberculosis (1999) and chronic hepatitis B (2003).

Physical examination at admission showed stable vital signs, multiple lymphadenopathies below $1 \mathrm{~cm}$ in laterocervical, submandibular and axillary region, lack of hepatoplenomegaly. The patient was afebrile at the time of presentation. The ramaining clinical examination was unremarkable. 
Table 1. Antiretroviral treatment for patient 2

\begin{tabular}{|c|c|c|c|c|c|c|}
\hline Time span & $1998-2000$ & $\begin{array}{c}\text { November 2002 - } \\
\text { August 2005 }\end{array}$ & $\begin{array}{c}\text { September 2005 - } \\
\text { January 2009 }\end{array}$ & $\begin{array}{c}\text { February 2009 - } \\
\text { April 2009 }\end{array}$ & $\begin{array}{c}\text { May 2009 - } \\
\text { September } \\
2014\end{array}$ & $\begin{array}{c}\text { September 2014 - } \\
\text { February 2016 }\end{array}$ \\
\hline Treatment & AZT+3TC & SQV+ABC+d4T & $\begin{array}{c}\text { SQV+RTV+ } \\
\text { ABC+d4T }\end{array}$ & $\begin{array}{c}\text { SQV+RTV+ } \\
\text { 3TC+ABC }\end{array}$ & $\begin{array}{c}\text { SQV+RTV+ } \\
\text { ABC/3TC }\end{array}$ & $\begin{array}{c}\text { DRV/RTV+ } \\
\text { EFV+3TC }\end{array}$ \\
\hline
\end{tabular}

$A Z T=$ zidovudin, $3 T C=$ lamivudin, $S Q V=$ saquinavir, $A B C=$ abacavir, $d 4 T=$ stavudin, $R T V=$ ritonavir, $D R V=$ darunavir, $E V R=$ efavirenz

Table 2. The evolution of CD4 lymphocytes count and HIV-RNA for patient 2

\begin{tabular}{|l|c|c|c|c|c|c|c|c|c|}
\hline & 1998 & 2000 & 2002 & 2006 & 2008 & 2009 & 2011 & 2014 & 2015 \\
\hline L CD4/mmc & 329 & 664 & 323 & 382 & 286 & 203 & 124 & 91 & 91 \\
\hline ARN-HIV (copies/ml) & - & - & - & 8200 & 16.700 & - & - & 13.700 & undetectable \\
\hline
\end{tabular}

LCD4=CD4 lymphocyte count

Laboratory tests on admission showed normal white blood cell count $(4170 / \mathrm{mmc})$, lymphopenia $(160 / \mathrm{mmc})$, severe normochromic microcytic anemia $(8 \mathrm{~g} / \mathrm{dl})$, normal number of platelets $(287,000 / \mathrm{mmc})$, biological inflammatory syndrome $(\mathrm{ESR}=78 \mathrm{~mm} / 1 \mathrm{~h}, \mathrm{CRP}=78.5$ $\mathrm{mg} / \mathrm{dl}$ ), slightly elevated LDH (486 U/l), normal liver enzymes. Quantiferon TB test was negative, as well as serum cryptococcal antigen. The discordant immunologic and virologic profile has been highlighted: CD4 lymphocyte count $=19$ cells/mmc and undetectable HIV-RNA. Other tests showed negative serology for Toxoplasma gondii (negative IgM and IgG antibodies), negative IgM antibodies and positive IgG antibodie for CMV, negative IgM antibodie for EBV (IgG antibodies not available), positive HBsAg, negative HBs antibodies, negative $\mathrm{HBeAg}$, positive $\mathrm{HBe}$ antibodies, negative IgM and IgG for HVD, negative antibody for HVC. Chest X-ray revealed no active pleural and pulmonary lesions.

On the fourt day of hospitalization, the patient developed fever, impaired general condition, aggravated cough, rapidly growing in size splenomegaly. The lymphadenopathies increased in size and new ones appeared in the supraclavicular region. He developed leucopenia, thrombocytopenia and elevated liver enzymes.

Following the new findings, the investigational profile was completed with a series of interdisciplinary consults and imaging investigations.

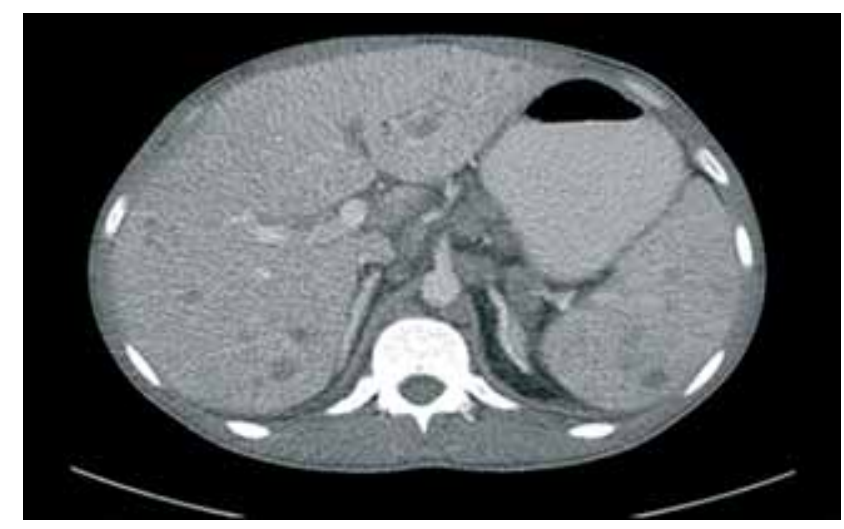

Figure 7. Abdominal CT scan patient 2. Multiple liver and spleen nodular lesions

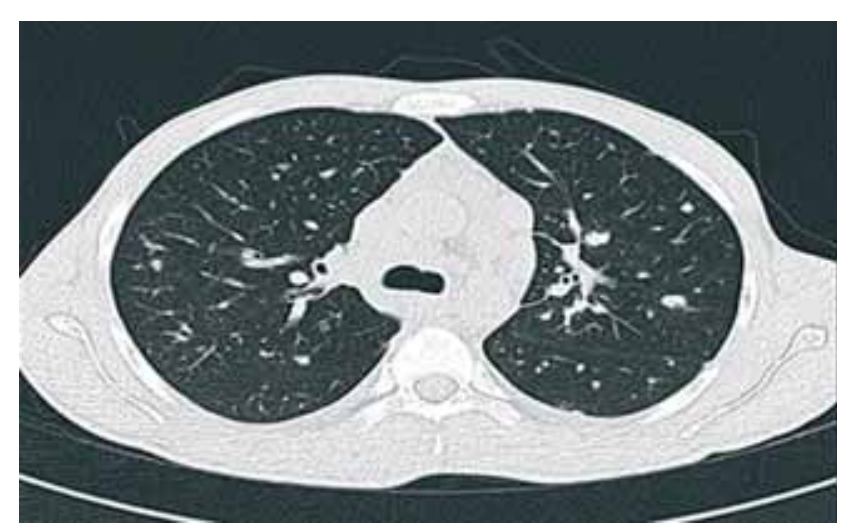

Figure 8. Thoracic CT scan patient 2. Multiple pulmonary unorganized nodular lesions

Abdominal ultrasound and CT scan (Fig. 7, 8) showed hepatomegaly $(23 \mathrm{~cm})$, splenomegaly $(18 / 6 \mathrm{~cm})$; multiple liver and spleen nodular images with maximum dimensions of $25 \mathrm{~mm}$ and multiple upper abdominal lymph nodes with maximum dimensions of $25 \mathrm{~mm}$.

Thoracic CT scan (Fig. 9) revealed mediastinal adenopathies with maximum dimensions of $27 \mathrm{~mm}$, multiple bilateral nodular images with sizes from 2 to $22 \mathrm{~mm}$ with unorganized 


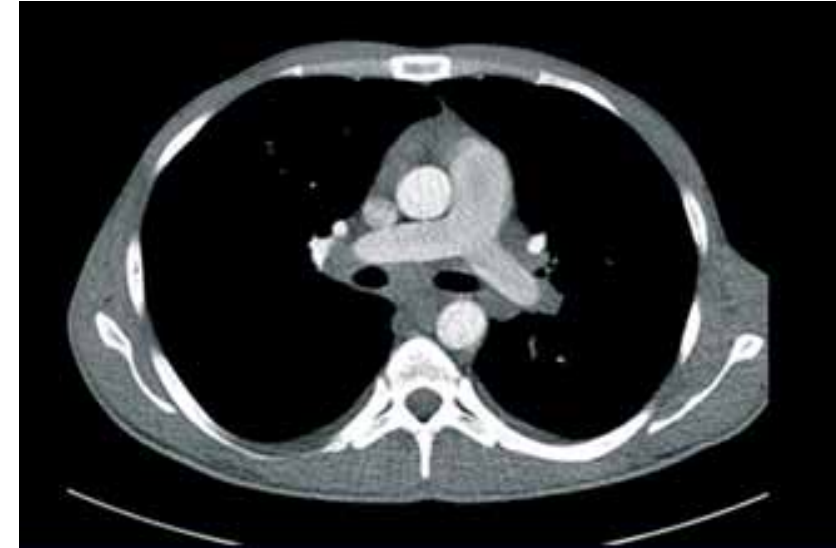

Figure 9. Thoracic CT scan patient 2. Mediastinal adenopathies

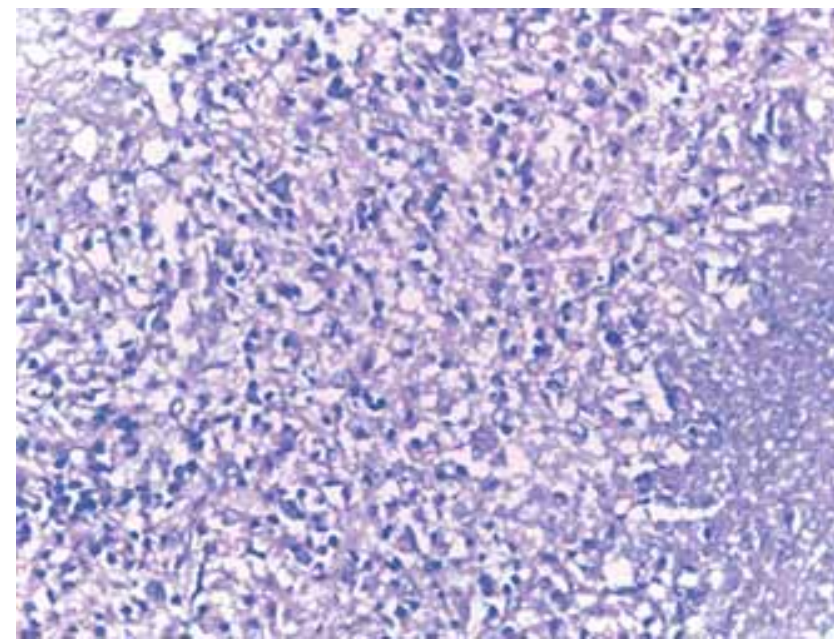

Figure 10. Histopathologic exam of pulmonary lesion patient 2. Tumoral Hodgkin/Reed-Sternberg cells, small reactice lymphocytes, histtiocytes, necrosis areas (Hematoxilyn-Eosin staining, objective 40x)

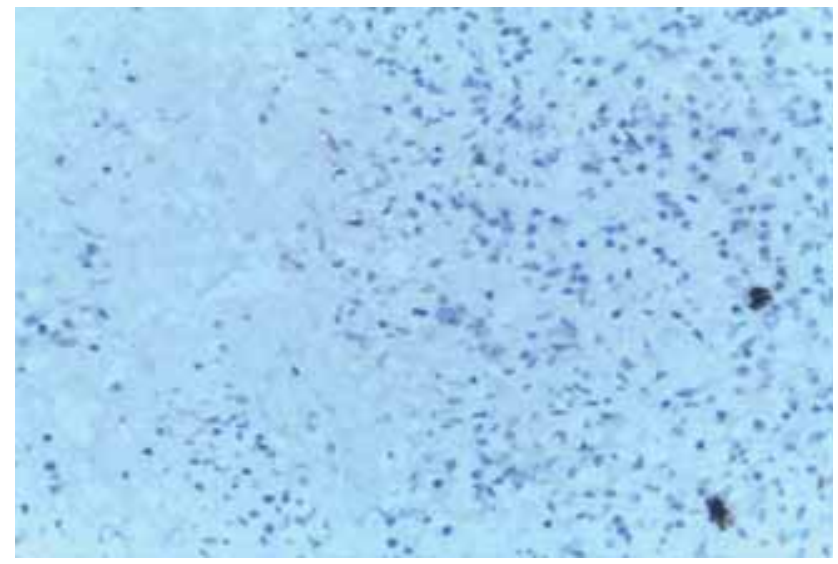

Figure 11. Immunohistochemitry for CD15 patient 2, objective $40 x$

distribution, minimum bilateral pleural efussions with $21 \mathrm{~mm}$ maximum thickness on the leftside and $17 \mathrm{~mm}$ on the rightside.

Cerebral MRI was normal.

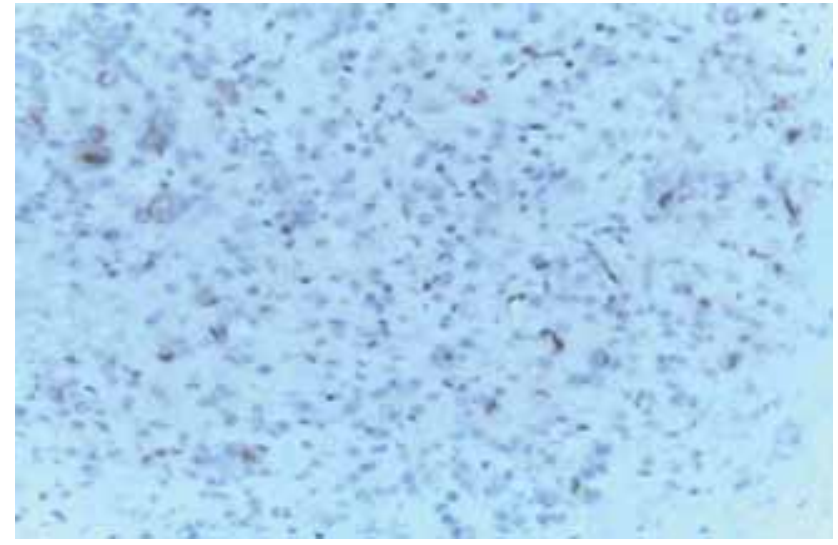

Figure 12. Immunohistochemitry for CD30 patient 2, objective $40 x$

Bronhoscopy showed a lesion of $2 / 2 \mathrm{~cm}$ confined to middle lobar bronchi from which biopsy was taken. Bacteriological exams of the collected respiratory pathological products were negative for bacteria, fungi and Koch bacillus.

Histopathology examinations performed to the lung biopsy revealed polymorphic tumor infiltration: mono- and binucleate Hodgkin/ ReddSternberg cells, small reactive lymphocytes, histiocytes, necrosis area. Immunohistochemistry (Figure 10,11,12) highlighted positive tumor cells for CD15, CD30 (Reed-Sternberg cell markers) and PAX5 (B marker). The panel was compatible with classic Hodgkin lymphoma.

Bone marrow biopsy was performed which showed no signs of marrow involvement.

The patient was hospitalized in our clinic for 14 days, during which he received antiretroviral treatment (Raltegravir, Emtricitabine, Tenofovir), prophylaxis for Pneumocystis carinii (trimethoprim-sulfamethoxazole) and Mycobacterium avium complex (clarithromycin), anti-tuberculosis medication (isoniazid, rifampicin, ethambutol, pyrazinamide - which was discontinued once we obtain the bacteriological results), corticosteroid (dexamethasone), gastric protector.

Following HL confirmation, the patient was transferred to hematology clinic, where was started chemotherapy (Doxorubicin, Bleomycin, Vinblastine, Dacarbazine). So far four cycles of chemotherapy have been performed, patients outcome being favorable. 


\section{DISCUSSION}

Both NHL and HL are hematologic pathologies with severe clinical and evolutive outcome in patients with HIV infection. The first type of lymphoma is classified among AIDS defining illnesses, while the second is not. Although both diseases are also found among patients without HIV infection, there are many epidemiological and outcome differences between the two groups. The incidence of these diseases is higher in HIV patients than in the general population. HL has a bimodal age distribuiton in the general population, with two peaks of incidence: 20-30 years and 50-65 years, while this kind of distribution dissapears in HIV patients, in whom most cases are recorded between the age 35-49 years (9). HIV associated NHL is correlated with older age (10) but among them, Burkitt lymphoma tends to be diagnosed in younger patients (11). The age of our presented patients was 27 years, less than the one reported in the literature.

CART led to a decrease in overall incidence of NHL. However, the incidence has remained stable for Burkitt's lymphoma subtype (12). In contrast, a "paradoxical" increase in incidence was recorded for HL (13). One explanation for this would be the role of Reed-Sternberg cells in producing factors that increase the influx of CD4 and inflammatory cells which, in return, provide signals that lead to neoplastic Reed-Sternberg cell proliferation. In severe immunosuppression conditions, this vicious circle is broken, and the progression of neoplastic cells is compromised (14). CART induced immune reconstruction and restored the described circuit, which could explain why there was no reduction in the incidence of HL in CART era. In addition, HIV associated HL is linked in most cases with the presence of the EBV virus, involved in the activation of pathways that promote the activation, growth and cell survival (9). Moreover, it was suggested the association between the risk of developing HL and the use of nucleoside reverse transcriptase inhibitors in CART (15). In our HL patient this class of medication was used for 16 months prior to his blood-cancer diagnosis.

Both types of lymphoma manifest more aggresively in HIV patients that in general population. HIV associated lymphoma is usually diagnosed in advanced stages, when severe extranodal involvement and B signs of the disease are present (16). This observations are applicable for our patients: they were both diagnosed with stage IV lymphoma and both had B signs of illness and extranodal involvement. Among frequent extranodal involvement of HIV associated NHL (gastrointestinal tract, liver, spleen, skin, central nervous system, bone marrow) (17), the first presented patient had liver, bone marrow and central nervous system involvement. Extranodal involvement is present in $70 \%$ of the cases of HIV associated HL, bone marrow being the most frequent (7). However, it was not demostrated in the case of the second patient. Other HL extranodal involvements in HIV patients are liver, skin and central nervous system. Unlike in general population, the incidence of mediastinal involvement is rare $13 \%$ vs $70 \%$ ) (18). However, it was present in our second patient, along with liver, spleen and lung involvement.

The duration and the level of immune-suppression is a predictive risk factor for the development of HIV associated NHL (19). The immune disorder induced by HIV and the reactivation of EBV due to poor control of T lymphocytes, leads to the stimulation and proliferation of B lymphocytes and NHL development. A low level of CD4 count is one of the unfavorable prognostic factors in these patients. However, Burkitt lymphoma tends to develop at a higher level of CD4 than other NHL subtypes (over 200 cells/mmc) (20). This observation was applicable for our patient also, who developed the disease at a CD 4 count of 442 cells/mmc. HIV associated HL is usually diagnosed at a CD4 count of $150-200$ cells/mmc (3). This observation is contrary to what we have found in our HL 
patient: his CD4 count was 19 cells/mmc. However, we must acknowledge the fact that lymphocytopenia is one of the common manifestations of HL, therefore a sudden decrease in CD4 count in a patient with a good adherence to CART and a previous good immune response, must draw attention towards the possible development of a hematologic pathology. The fact that the patient had undetectable HIV-RNA certifies this observation.

Unfortunately, the prognosis of HIV associated lymphoma is still a poor one. Over the time, some prognostic factors were proposed. In preCART era, they were mainly immunologic factors (CD4 count), but nowadays they are more similar to those used in general population, being based on International Prognostic Index (IPI) used in non-HIV patients with aggressive NHL. IPI sums up patient's age, LDH level, performance level, the stage of hematologic disease, the number of extranodal involvements. In HIV positive patients, the CD4 lymphocytes count is added to the previous factors (21). The prognostic index for both patients was 4 , which signifies a poor prognosis and a survival rate of $15 \%$ at 1 year. Although both patients had a similar number of poor prognostic factors and the same prognostic index, only one of them rapidly evolved towards death: the one with Burkitt-like NHL with a high CD4 count but detectable HIV-RNA, whereas the one with undetectable HIV-RNA survived and is responding well to chemotherapy. Therefore, apart from the histological type of the lymphoma and the level of CD4 count, we can speculate that the level of control over the viral replication is an important determinant of the survival rate and prognosis of these patients. 0 good viral suppression could be a positive factor for the patient's outcome. In the deceased patient we found a two year medical history of discordant HIV serology tests, so it is very likely that the patient had HIV infection for at least that period of time without benefiting from CART that would have controlled the viral replication.
The most important element that contributed to the improvement of the evolution of the patients with HIV-associated lymphoma that underwent chemotherapy was CART. It allowed the use of potent chemotherapy protocols, similar with those used in general population, thus becoming an important positive prognostic factor for the patients with HIV associated lymphoma (22). Therefore it's important for these patients to be treated with CART with high potency, good tolerability and very little interactions with chemotherapeutic agents. Among antiretroviral drugs, zidovudine is relatively contraindicated because of its medullar suppressive effect. Protease inhibitors, especially ritonavir, have effect on p450 cytochrome, which enhances the toxicity of chemotherapeutic agents. In contrast, CART that include integrase inhibitors have few drug interactions, are potent and well tolerated, being an appropriate choice for HIV patients who undergo aggressive lymphoma treatment protocols. For our patients we have chosen such a CART regimen that included raltegravir, emtricitabine, tenofovir.

The differential diagnosis of HIV associated lymphoma is a broad one. Tuberculosis, invasive fungal infections, toxoplasmosis are among the most frequent pathologies brought into question in such a context. Sometimes the patient is started on empiric treatment for these infectious pathologies until the investigations establish the definitive and accurate diagnosis.

In this respect, we must emphasize other important issues raised by the investigational and therapeutic management of HIV associated lymphoma, namely, the need to involve multiple medical specialists. A good and close interdisciplinary collaboration is mandatory. In the case of our patients such a multidisciplinary team included specialists in infectious diseases, hematology, lung diseases, histopathology, microbiology, radiology. Coordination of joint efforts and continuous communication between the members of the multidisciplinary team is the key to a fast and accurate diagnosis. 


\section{CONCLUSIONS}

HIV associated lymphoma may embrace different clinical aspects. They have to be included into the differential diagnosis panel in patients who present with sudden rapid growth of lymph nodes, neurologic manifestations, discordant immunologic and virologic response despite a good CART adherence.

\section{REFERENCES}

1. Shiels M.S., Pfeiffer R.M., Gail M.H. et al. Cancer burden in HIV-infected population in the United States. Journal of the National Cancer Institute. 2011;9:753-62

2. Robbins H.A., Shiels M.S., Pfeiffer R.M., Engels E.A. Epidemiologic contributions to recent cancer trends among HIV-infectected people in the United States. AIDS 2014;28(6):881-890

3. Biggar R.J., Jaffe E.S., Goedert J.J., Chaturvedi A., Pfeiffer R., Engels E.A. Hodgkin lymphoma and immunodeficiency in persons with HIVIAIDS. Blood 2006. 108(12):3786-91

4. Herida M., Mary-Krause M., Kaphan R. et al. Incidence of non-AIDS defining cancers before and during the highly active antiretroviral therapyera in a cohort oh human immunodeficiency virus-infected patients. J.Clin. Oncol. 2003;21:3447-3453

5. Rabkin C.S. Epidemiology of AIDS-related malignancies. Curr Opin Oncol. 1994;6(5):492-6

6. Biggar R.J., Curtis R.E., Cote T.R., Rabkin C.S., Melbye M. Risk of other cancers following Kaposi's sarcoma: relation to acquired immunodeficiency syndrome. Am J Epidemiol. 1994.15;139(4):362-8

7. Berrenguer J., Miralles P., Ribera J.M. et al. Characteristics and outcome of AIDS-related lymphoma before and after the introduction of highly active antiretroviral therapy. J. Acquir. Immune Defic. Syndr. 2008;47:422-428

8. Behler C.M., Kapla L.D. Advances in management of HIV-related non-Hodgkin lymphoma. Curr. Opin. Oncol. 2006;18(5):437-43

9. Dolcetti R., Bolocchi M., Gloghini A., Carbone A. Pathogenic and histogenic features of HIV-associated Hodgkin's lymphoma disease. Eur J Cancer. 2001; 37(10):1276-1287

10. Bohlius J., Schmidlin K., Costagliola D. et al. Incidence and risk factors of HIV-related non-Hodgkin's lymphoma in the era of combination antiretroviral therapy: a European multicohort study. Antivir Ther. 2009; 14(8): 1065-1074

11. Beral V., Peterman T., Berkelman R. et al. AIDS-associated non-Hodgkin lymphoma. Lancet 1991;337:805-9
Unlike in general population, HIV associated lymphoma have more aggressive aspects, extensive extranodal involvement and a worse prognostic.

Diagnostic and therapeutic management of HIV associated lymphoma involves extensive investigations and the active collaboration of a multidisciplinary team.

12. International Collaboration on HIV and cancer. Highly active antiretroviral therapy and incidence of cancer in human immunodeficiency virus infected adults. J Natl Cancer Inst. 2000; 92: 1823-1830

13. Spina M., Gloghini A., Carbone A. et al. Hodgkin's Disease in Patients with HIV Infection. Advances in Hematology. vol. 2011, Article ID 402682, 7 pages, 2011. doi:10.1155/2011/402682

14. Gloghini A., Carbone A., "Why would the incidence of HIV-associated Hodgkin lymphoma increase in the setting of improved immunity?" International Journal of Cancer, 2007; 12(120):2753-2754

15. Powls T., Robinson D., Stebbing J. et al. Highly active antiretroviral therapy and the incidence of non-AIDS defining cancers in people with HIV infection. J. Clin. Oncol. 2009; 27: 884-890

16. Lee B., Bower M., Newsome-Davis T., Mark N. HIV-relatedlymphoma. HIV Ther. 2010; 4(6):649-659

17. loachim H. Lymphoma: an opportunistic neoplasm of AIDS. Leukemia. 1992; 6 (suppl 3): 65-95

18. Andrieu J.M., Roithmann S., Tourani J.M. Hodgkin's disease during HIV1 infection: the French registry experience. French registry of HIV-associated tumors. Ann. Oncol. 1993; 4: 635-641

19. Grulich A.E., Wan X., Law M.G. et al. B-cell stimulation and prolonged immune deficiency are risk factors for non-Hodgkin's Iymphoma in people with AIDS. AIDS. 2000; 14: 133-140

20. Gates A.E., Kaplan L.D. Biology and management of AIDS-associated non-Hodgkin's lymphoma. Hematol Oncol Clin North Am. 2003; 17: 821-841

21. Bower M., Gazzard B., Mandalia S. et al. A prognostic index for systemic AIDS-related non-Hodgkin's lymphoma treated in the era of highly active antiretroviral therapy. Ann. Intern. Med. 2005; 143: 265-273

22. Besson C., Goubar A., Gabarre J. et al. Changes in AIDS-related lymphoma since the era of highly active antiretroviral therapy. Blood. 2001;98:2339-44 